\title{
BEAMFORMING AND PAPR REDUCTION FOR MISO-OFDM SYSTEMS
}

\author{
Jingon Joung, Eui-Rim Jeong, and Yong H. Lee \\ School of Electrical Engineering and Computer Science \\ Korea Advanced Institute of Science and Technology (KAIST) \\ 373-1 Guseong-dong, Yueong-gu, Daejeon, Republic of Korea 305-701 \\ e-mail: \{jgjoung, july\}@stein.kaist.ac.kr; yohlee@ee.kaist.ac.kr
}

\begin{abstract}
A peak-to-average power ratio (PAPR) reduction technique for multiinput single-output (MISO) orthogonal frequency division multiplexing (OFDM) systems with beamforming is proposed. This technique consists of adaptive antenna selection and phase rotation. By using the proposed method, a large reduction in PAPR can be obtained at the expense of a small signal-to-noise ratio (SNR) loss. According to the simulation results, the PAPR is reduced by $10 \%$ in terms of complementary cumulative distribution function (CCDF) with negligible SNR loss. If a $1 \mathrm{~dB}$ SNR loss is allowed, a $33 \%$ reduction in PAPR can be achieved.
\end{abstract}

Index Terms-Orthogonal frequency division multiplexing (OFDM) systems, peak-to-average power ratio (PAPR), multipleinput single-output (MISO), beamforming.

\section{INTRODUCTION}

Multiple-input single-output (MISO) systems, which have multiple antennas at the transmitter and a single antenna at the receiver, provide spacial diversity, which can reduce the received signal power fluctuations in fading environments [1]. Among techniques achieving maximum diversity gain, transmit beamforming $(\mathrm{BF})$ is a simple approach when the channel state information (CSI) is available at the transmitter. In the BF systems, the burden of the CSI fed back from the receiver to the transmitter, when the up/down link channels are not reciprocal, can be greatly reduced by using a codebook [2]. The $\mathrm{BF}$ techniques for narrow band channels can be directly extended to wide band frequency-selective channels by using frequency division multiplexing (OFDM) [3].

The combination of MISO and OFDM (MISO-OFDM) is an attractive transmission technique in fading environments. However, the potentially high peak-to-average ratio (PAPR) of the MISOOFDM signals may lead to inband signal distortion and inter-channel interference when using a typical RF power amplifier without sufficient back-off from its saturation point. Therefore, PAPR reduction for MISO-OFDM signals is an important issue for high power efficiency, low power consumption, and low manufacturing cost. There have been many studies on PAPR reduction in the literature, but most of them were developed for single-input single-output (SISO) OFDM signals [4]-[7].

In this paper, we consider a PAPR reduction method for the MISO-OFDM system employing a BF technique. Among the ex-

This work was supported in part by the University Information Technology Research Center (ITRC) Program of the Korean government and Brain Korea 21 Project, The school of information technology, KAIST in 2007. isting PAPR reduction methods for SISO-OFDM signals, phase rotation (PR) methods in the category of the partial transmit sequences (PTS) [4]-[7] can be easily applied to MISO-OFDM signals without any loss of signal-to-noise ratio (SNR) at the receiver because of the phase-invariant characteristic of the $\mathrm{BF}$ vector [2]. Although it is observed that the existing PTS method itself can effectively reduce the PAPR of the MISO-OFDM signals, we propose a new antenna selection (AS) and BF scheme to reduce the PAPR further.

In the proposed scheme, the total subcarriers are divided into small groups, and each group can select a few of the transmit antennas for data transmission while the conventional $\mathrm{BF}$ system always uses all of the antennas. Unless all of transmitter antennas are used for the BF, a certain amount of SNR loss is inevitable. However, by determining the AS scheme properly at each group and employing the $\mathrm{PR}$, a great reduction in PAPR can be achieved while restricting the SNR loss to a small amount. We propose an AS and PR rule that can make such compromise between the minimization of the PAPR and the maximization of the effective channel gain at the worst subcarrier group. According to the simulation results, the proposed method can reduce $10 \%$ PAPR by about $0.5 \mathrm{~dB}(10 \%)$ with a negligible SNR loss. If $1 \mathrm{~dB}$ SNR loss is allowed, $1.7 \mathrm{~dB}(33 \%)$ reduction in PAPR can be achieved. For the proposed AS and PR, a BF vector and corresponding channel gain at each BF group should be fed back from the receiver to the transmitter. However, the receiver does not require any additional side information except $P R$ values for PAPR reduction, which is the same information in the conventional PR schemes.

This paper is organized as follows. Section 2 presents the proposed system model, and an AS and PR rule is proposed in Section 3. The PAPR and bit error rate (BER) performances of the proposed method are demonstrated through computer simulation in Section 4. Finally, we conclude in Section 5.

\section{PROPOSED SYSTEM MODEL}

The system model considered in this paper is shown in Fig. 1. The transmitter and receiver have $N_{T}$ and 1 antennas, respectively. The MISO-OFDM symbol has a total of $N$ subcarriers. The main features of the proposed system model can be characterized by AS, BF, and PR.

Antenna selection $(A S)$ : Define the frequency domain MISO channel $\mathbf{h}(n)$ as $\left[h_{1}(n), \ldots, h_{N_{T}}(n)\right]^{T} \in \mathbb{C}^{N_{T} \times 1}$, where $h_{l}(n)$ denotes the complex channel gain between the $l$-th transmit antenna and the receive antenna for $n$-th subcarrier. It is assumed that $\left\{h_{l}(n), l=\right.$ $\left.1, \ldots, N_{T}\right\}$ are independent, identically distributed (i.i.d) Gaussian with a mean of zero and a variance of 1 . In our system model, con- 


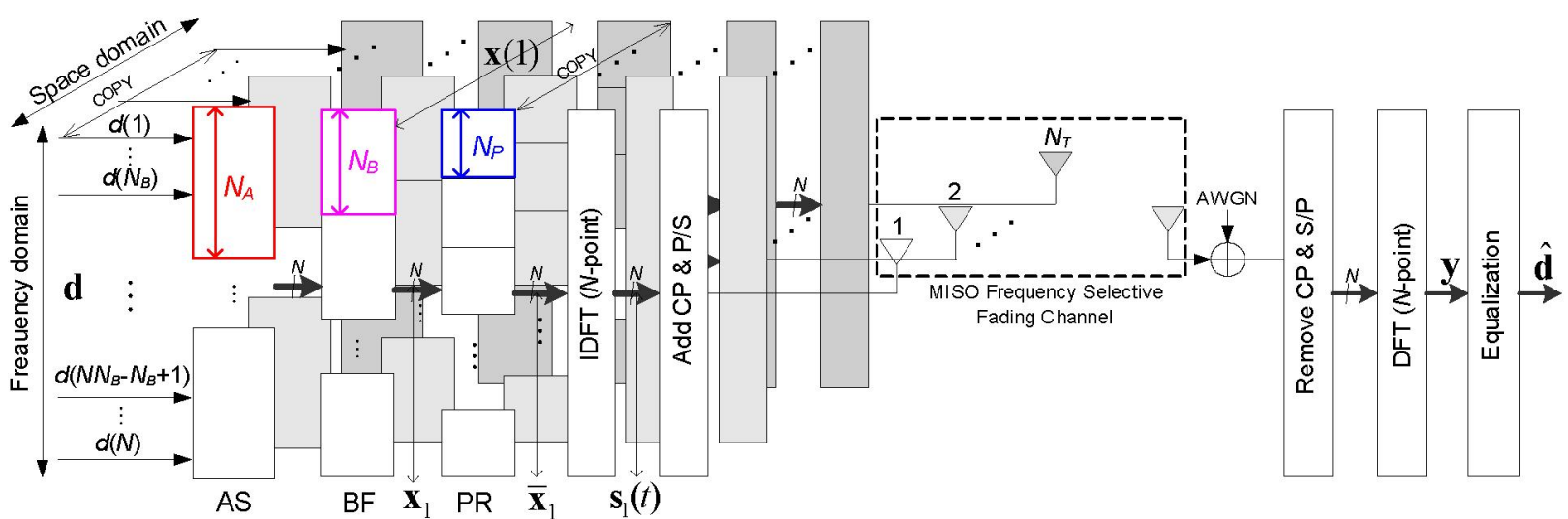

Fig. 1. The proposed MISO-OFDM system with beamforming.

secutive $N_{A}$ subcarriers comprise one AS group. Since the total number of subcarriers is $N$, there are $N / N_{A}$ AS groups. Each AS group can use $M\left(M \leq N_{T}\right)$ to $N_{T}$ transmit antennas for data transmission. Therefore, the number of possible AS combinations at each group is $\sum_{m=M}^{N_{T}} N_{T} \mathrm{C}_{\mathrm{m}}$. If we denote the $q$-th AS matrix set as $\boldsymbol{\Gamma}_{q}=\left\{\boldsymbol{\Gamma}_{q}(1), \cdots, \boldsymbol{\Gamma}_{q}(N)\right\}$, where $\boldsymbol{\Gamma}_{q}(n)$ is a AS matrix for $n$ th subcarrier, the modified channel vector $\overline{\mathbf{h}}_{q}(n)$ by the $A S$ can be written as

$$
\overline{\mathbf{h}}_{q}^{T}(n)=\mathbf{h}^{T}(n) \boldsymbol{\Gamma}_{q}(n), 1 \leq q \leq Q,
$$

where $\boldsymbol{\Gamma}_{q}(n) \in \mathbb{R}^{N_{T} \times N_{T}}$ is a diagonal matrix whose $l$-th element is 1 if $l$-th antenna is used for transmission and 0 otherwise. Therefore, the number of 1 in $\Gamma_{q}(n)$ is the same as the number of the selected antennas by the AS. Note that $\Gamma_{q}(n)$ is identical in an AS group, i.e., $\boldsymbol{\Gamma}_{q}\left(N_{A}(k-1)+1\right)=\cdots=\Gamma_{q}\left(N_{A} k\right)$ for $k=1, \ldots, N / N_{A}$. Considering all of the subcarriers, $1 \leq n \leq N$, the total number of AS combinations becomes $Q=\left(\frac{N}{N_{A}}\right)^{\sum_{m=M}^{N_{T}} N_{T} C_{m}}$.

Beamforming $(B F)$ : In MISO systems, each subcarrier transmits one stream $d(n)$. In AS procedure, $d(n)$ is copied to the transmit antennas for BF, which is denoted by a vector $\mathrm{d}(n) \in \mathbb{C}^{N_{T} \times 1}$ and multiplied by a $\mathrm{BF}$ vector $\mathbf{w}(n) \in \mathbb{C}^{N_{T} \times 1}$. The $\mathrm{BF}$ output can be written as

$$
\mathbf{x}(n)=\mathbf{w}(n) \circ \mathbf{d}(n),
$$

where $\circ$ is the element-wise Schur matrix product [9]. Here, to reduce the feedback information, we also assume that the $B F$ vector is the same in a BF group and that each $\mathrm{BF}$ group has $N_{B}$ subcarriers, and thus there are a total of $N / N_{B}$ beamformers. Therefore, $\mathbf{w}\left(N_{B}(k-1)+1\right)=\cdots=\mathbf{w}\left(N_{B} k\right)$ for $k=1, \ldots, N / N_{B}$. To maximize the received SNR, the BF vector $\mathrm{w}(n)$ is determined using the singular value decomposition (SVD) of the effective channel vector. Since the effective channel of our system is not $\mathbf{h}_{q}(n)$, but $\overline{\mathbf{h}}_{q}(n)$ followed by AS, the SVD should be done on $\overline{\mathbf{h}}_{q}(n)$. Further, to determine one BF vector, a representative channel vector in a BF group should be defined. Let $\hat{\mathbf{h}}_{q}(n)$ be a representative channel vector in a $\mathrm{BF}$ group to which subcarrier index $n$ belongs. Each $\mathrm{BF}$ group has one representative channel vector, i.e., $\hat{\mathbf{h}}_{q}\left(N_{B}(k-1)+1\right)=\cdots=\hat{\mathbf{h}}_{q}\left(N_{B} k\right)$ for $k=1, \ldots, N / N_{B}$. By SVD, $\hat{\mathbf{h}}_{q}(n)$ can be decomposed as

$$
\hat{\mathbf{h}}_{q}^{T}(n)=1 \cdot\left[\hat{\sigma}_{q}(n) 0 \cdots 0\right] \cdot \hat{\mathbf{V}}_{q}^{H}(n),
$$

where $\hat{\sigma}_{q}(n)$ is a singular value of $\hat{\mathbf{h}}_{q}^{T}(n)$ and $\hat{\mathbf{V}}_{q}(n) \in \mathbb{C}^{N_{T} \times N_{T}}$ is a right singular matrix of $\hat{\mathbf{h}}_{q}^{T}(n)$. The BF vector $\mathbf{w}(n)$ is given by the first column vector of $\hat{\mathbf{V}}(n)$ and a set of $B F \mathbf{w}=\{\mathbf{w}(1), \ldots, \mathbf{w}(N)\}$ After BF for all BF groups, the frequency-domain OFDM symbol at $l$-th antenna can be written as

$$
\mathbf{x}_{l}=\left[x_{l}(1), \ldots, x_{l}(N)\right]^{T} \in \mathbb{C}^{N \times 1},
$$

where $x_{l}(n)$ is the $l$-th element of $\mathbf{x}(n)$ in (2). According to AS, if the $l$-th transmit antenna was not selected, then the $l$-th element of a vector $\mathrm{w}(n)$ becomes zero and $x_{l}(n)$ in (4) also becomes zero. Consequently, there are more degree of freedom for mitigating the PAPR.

Phase rotation (PR): After AS and BF, $\mathrm{PR}$ is performed on all subcarriers at each antenna with $\mathrm{x}_{l}$ in (4). The PR output can be written as

$$
\overline{\mathbf{x}}_{l}=\boldsymbol{\Theta} \mathbf{x}_{l}, \text { for } l=1, \ldots, N_{T},
$$

where $\Theta \in \mathbb{C}^{N \times N}$ is a diagonal matrix whose $n$-th element $e^{j \theta(n)}$ represents the phase rotation at $n$-th subcarrier. All antennas use the identical $\Theta$. By choosing appropriate $\Theta$, the PAPR can be effectively reduced. As groups for $\mathrm{AS}$ and $\mathrm{BF}$, all of the subcarriers are divided into PR groups. Assuming that a PR group has $N_{P}$ consecutive subcarriers, the diagonal elements of $\Theta$ have the following property: $e^{j \theta\left(\left(N_{P}-1\right) k+1\right)}=\cdots=e^{j \theta\left(N_{P} k\right)}$, for $k=1, \ldots, N / N_{P}$. After PR, the time domain signal at $l$-th antenna is obtained by IDFT (inverse DFT):

$$
\mathbf{s}_{l}=\mathbf{F}^{H} \overline{\mathbf{x}}_{l},
$$

where $\mathbf{F}^{H} \in \mathbb{C}^{N \times N}$ is the IDFT matrix whose $(a, b)$-th element is $\frac{1}{\sqrt{N}} e^{j 2 \pi(a-1)(b-1) / N}$. The PAPR of the $l$-th transmit antenna is defined as

$$
P A P R_{l}(\boldsymbol{\Gamma}, \mathbf{w}, \boldsymbol{\Theta})=\frac{\max \left(\left|s_{l}(i)\right|^{2}\right)}{P_{T} / N_{T}}, i=1, \ldots, N,
$$

where $s_{l}(i)$ is the $i$-th element of $\mathbf{s}_{l}, \max (\cdot)$ is the maximum, and $P_{T}$ is the total transmission power.

\section{ANTENNA SELECTION AND PHASE ROTATION METHOD}

We consider the $A S$ and $P R$ in the $B F$ systems to minimize the PAPR and maximize the minimum effective channel gain, simultaneously. Thus, the feedback information for effective channel gain 
is required. For a given $\mathrm{AS}$ scheme, the $\mathrm{BF}$ vector can be calculated. The PR also follows from AS. Thus, the BF vector and PR are as various as the AS combinations. An important observation is made that the PAPR and the minimum channel gain cannot be improved at the same time. Hence, this section proposes a general rule for finding AS, providing a trade-off between them.

\subsection{Antenna Selection (AS)}

As mentioned in Section 2, the AS $\boldsymbol{\Gamma}_{q}$ over all subcarriers has $Q$ different kinds of sets. First, a certain AS $\boldsymbol{\Gamma}_{q}=\left\{\boldsymbol{\Gamma}_{q}(1), \cdots, \boldsymbol{\Gamma}_{q}(N)\right\}$ is chosen before $\mathrm{BF}$ and $\mathrm{PR}$.

\subsection{Beamforming (BF)}

$\mathrm{BF}$ determines the effective channel gain, i.e., SNR with previously fixed AS $\Gamma_{q}$. The objective of the proposed BF is maximizing the minimum channel gain among $N$ subcarriers. For a given $\mathrm{AS}$ set $\Gamma_{q}$, the minimum effective channel gain is defined as

$$
G_{\min }\left(\boldsymbol{\Gamma}_{q}\right)=\min _{n=1, \ldots, N} \sigma_{\Gamma_{q}}(n),
$$

where $\sigma_{\Gamma_{q}}(n)$ represent the singular value in (3) when the AS scheme is $\Gamma_{q}$.

\subsection{Phase Rotation (PR)}

$\mathrm{PR}$ follows $\mathrm{AS}$ and $\mathrm{BF}$ and does not change the effective channel gain. The PR matrix is designed according to transmitted symbol vector $\mathbf{x}_{l}$ in (4), $\mathrm{AS}$ and $\mathrm{BF}$ matrices. However, the $\mathrm{BF}$ vector totally depends on $\mathrm{AS} \boldsymbol{\Gamma}_{q}$, and it can be seen that PR is determined by AS only. For a given $\boldsymbol{\Gamma}_{q}$, we define the achievable minimum PAPR of the worst antenna which has largest PAPR,

$$
P_{\min }\left(\boldsymbol{\Gamma}_{q}\right)=\min _{\Theta} P A P R_{\max _{l}}\left(\boldsymbol{\Gamma}_{q}, \mathbf{w}, \boldsymbol{\Theta}\right)
$$

by using PR $\Theta$, where $P A P R_{\max _{l}}\left(\boldsymbol{\Gamma}_{q}, \mathbf{w}, \Theta\right)$ represents the largest PAPR among $N_{T}$ antennas for given $\boldsymbol{\Gamma}_{q}, \mathbf{w}$, and $\boldsymbol{\Theta}$. Also, the PR set $\Theta$ that result in $P_{\text {min }}$ is defined as $\Theta_{\text {min }}$. Since solving (9) for $\Theta$ is very complex and time-consuming, the existing works confined the possible phase rotation values into a finite set. This paper uses a finite phase set $\{0, \pi / 2, \pi, 3 \pi / 2\}$, which is widely used [5].

\subsection{Proposed AS-PR-BF System}

It turns out that maximizing $G_{\min }\left(\boldsymbol{\Gamma}_{q}\right)$ in (8) and minimizing $P_{\min }\left(\boldsymbol{\Gamma}_{q}\right)$ cannot be achieved simultaneously. Hence, we propose a general AS rule that can make a compromise between these two.

$$
\boldsymbol{\Gamma}_{\max }=\underset{\boldsymbol{\Gamma}_{q}, q=1, \ldots, Q}{\arg \max }\left(\frac{\lambda}{P_{\min }\left(\boldsymbol{\Gamma}_{q}\right)}+(1-\lambda) G_{\min }\left(\boldsymbol{\Gamma}_{q}\right)\right),
$$

where $\lambda$ is a positive real value between 0 and 1 . Once $\Gamma_{\max }$ is obtained, the BF vector and PR matrix can be found from (3) and (9), respectively. Equations (8) and (9) are combined in (10). If $\lambda=1$, the antenna selection is done to minimize PAPR only, whereas if $\lambda=0$, the antenna selection maximizes SNR only. Therefore, $\lambda$ determines the trade-off between these two extreme cases.

\subsection{Summary of Scenario}

In the proposed AS-PR-BF system, the receiver feeds back the desired $\mathrm{BF}$ vector or corresponding codebook index to the transmitter, and the channel gain should be also fed back to the transmitter (see Subsection 3.2). In the BF system based on the codebook, the receiver usually feeds back the phase $\phi$ rotated BF vector to the transmitter in the form of the codebook index. Since the receiver knows the phase $\phi$ exactly, the receiver counter-rotates the received signal before performing detection, and any BF vectors associated with the AS combinations can be generated at the transmitter since the transmitter knows both of the phase rotated $\mathrm{BF}$ vector, which does not perform $\mathrm{AS}$, and the effective channel gain. Furthermore, all the new AS-PR-BF vectors have the same phase rotation value $\phi$ as the original BF vector. This implies that the receiver does not need the AS information for detection, so that no additional side feed forward information on the AS is required at the receiver. However, similarly to the conventional PR-BF systems, the PR $\Theta$ should be fed forward to the receiver.

\section{SIMULATION RESULTS}

Computer simulations were conducted to examine the performance trade-off between PAPR and BER. The complementary cumulative distribution function (CCDF) of PAPR and the average BER were evaluated. The $\mathrm{CCDF}$ is defined as follows [8]:

$$
C C D F\left(P A P R_{0}\right)=\operatorname{Pr}\left(P A P R>P A P R_{0}\right) .
$$

In the simulations, the MISO channel is obtained by generating independent Gaussian random variables with a mean of zero, and the results shown below are the averages over 1,000 independent trials. The simulation parameters are shown in Table 1. The eigen BF for a group uses the representative channel or BF vector, which has the largest instantaneous channel gain.

Figs. 2 and 3 show the CCDF of PAPR and BER, respectively. In these figures, the conventional BF did not use any PAPR reduction schemes. When $\lambda$ is 0 , the proposed AS-PR focuses on maximizing the effective channel gain; therefore, all transmit antennas were selected, i.e., PR without AS. The result indicates that about a $2 \mathrm{~dB}$ reduction in PAPR can be achieved by PR only without any BER performance loss. Also, the proposed method obtained further PAPR reduction compared to that of the system using PR only, at the expense of BER performance. Let the PAPR reduction gain at $10 \%$ CCDF and SNR loss at $1 \%$ BER be $G_{P A P R}$ and $L_{B E R}$, respectively. Both of $G_{P A P R}$ and $L_{B E R}$ depend on $\lambda$. As expected from (10), when $N_{P}$ and $N_{A}$ are fixed, $G_{P A P R}$ and $L_{B E R}$ increase as $\lambda$ increases. For $\lambda=0.7, G_{P A P R} \approx 0.5 \mathrm{~dB}(10 \%)$ with negligible $L_{B E R}$. In contrast, for $\lambda=0.92, G_{P A P R} \approx 1.7 \mathrm{~dB}(33 \%)$ while $L_{B E R} \approx 1 \mathrm{~dB}$. These results indicate that by adjusting $\lambda$, some different trade-offs between $G_{P A P R}$ and $L_{B E R}$ can be obtained.

\section{CONCLUSION}

A dynamic trade-off between PAPR and BER using the proposed transmit antenna selection and the phase rotation method for beamforming MISO-OFDM systems was examined to reduce PAPR while providing a comparable BER performance to the conventional beamforming systems. Examining the trade-off between PAPR gain and BER gain in various parameters and finding the optimal trade-off point remain as topics for future study. 
Table 1. Simulation Parameters

\begin{tabular}{|c|c|}
\hline Parameter & Values \\
\hline FFT size & 128 \\
\hline \# of used subcarriers $N$ & 126 \\
\hline \# of channel taps & 2 \\
\hline$\#$ of transmit antennas $\left(N_{T}\right)$ & 3 \\
\hline$\#$ of receive antennas $\left(N_{R}\right)$ & 1 \\
\hline$\#$ of selection antennas $(M)$ & 2 \\
\hline AS groups $\left(N_{A}\right)$ & 21 subcarriers \\
\hline Selection $\left(\Gamma_{q}(n)\right)$ & {$\left[\begin{array}{lll}1 & 0 & 0 \\
0 & 1 & 0 \\
0 & 0 & 1\end{array}\right],\left[\begin{array}{lll}1 & 0 & 0 \\
0 & 1 & 0 \\
0 & 0 & 0\end{array}\right],\left[\begin{array}{lll}1 & 0 & 0 \\
0 & 0 & 0 \\
0 & 0 & 1\end{array}\right],\left[\begin{array}{lll}0 & 0 & 0 \\
0 & 1 & 0 \\
0 & 0 & 1\end{array}\right]$} \\
\hline \# of OFDM symbols & 1,000 \\
\hline $\mathrm{BF}$ groups $\left(N_{B}\right)$ & 42 subcarriers \\
\hline Beamformer & eigen $B F$ \\
\hline PR groups $\left(N_{P}\right)$ & 42 subcarriers \\
\hline $\operatorname{PR}\left(e^{j \theta}\right)$ & $1,-1, j,-j$ \\
\hline Modulation & QPSK \\
\hline Tx power $\left(P_{T}\right)$ & 1 \\
\hline Equalizer (EQ) & zero-forcing linear EQ \\
\hline
\end{tabular}

\section{REFERENCES}

[1] D. Tse and P. Viswanath, Fundamentals of Wireless Communication, 1st ed., Cambridge univ. press, 2005.

[2] D. J. Love, R. W. Heath, Jr., and T. Strohmer, "Grassmannian beamforming for multiple-input multiple-output wireless systems," IEEE Trans. Inform. Theory, vol. 49, no. 10, pp. 27352747, Oct. 2003.

[3] J. Choi and R. W. Heath, Jr., "Interpolation based transmit beamforming for MIMO-OFDM with limited feedback," IEEE Trans. Signal Processing, vol. 53, no. 11, pp. 4125-4135, Nov. 2005.

[4] S. H. Muller and J. B. Huber, "OFDM with reduced peak-toaverage power ratio by optimum combination of partial trnasmit sequences," Electron. Lett., vol. 33, no. 5, pp. 368-369, Feb. 1997.

[5] L. J. Cimini, Jr. and N. R. Sollenberger, "Peak-to-average power ratio reduction of an OFDM signal using partial transmit sequences," IEEE Commun. Lett., vol. 4, no. 3, pp. 86-88, Mar. 2000.

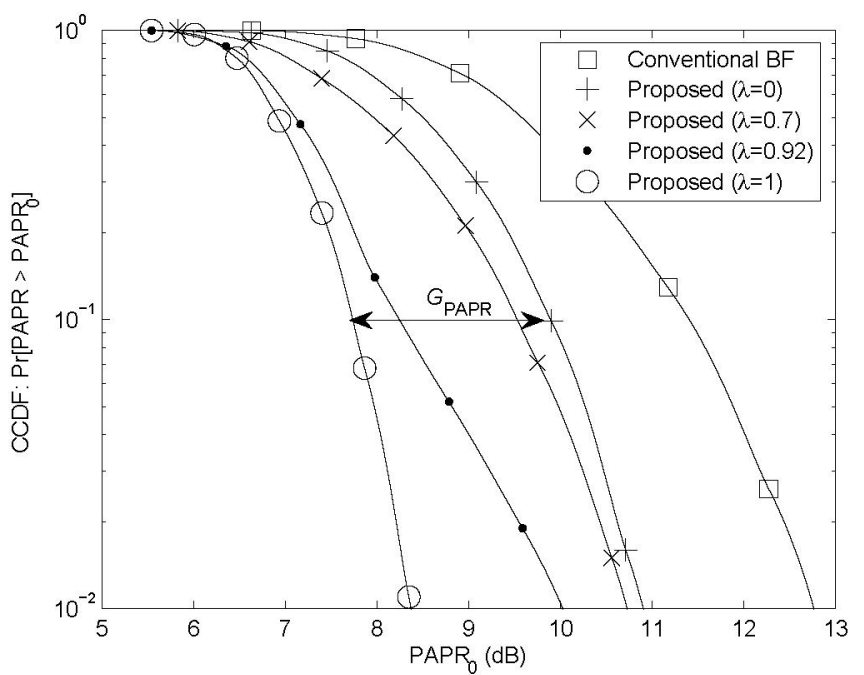

Fig. 2. PAPR performance

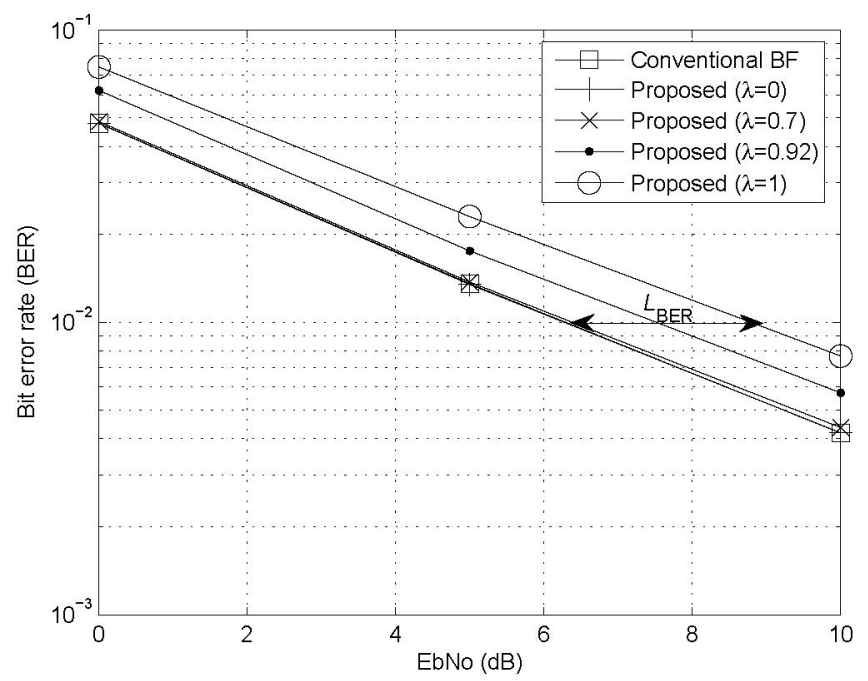

Fig. 3. BER performance

[6] C. Tellambura, "Improved phase factor computation for the PAR reduction of an OFDM signal using PTS," IEEE Commun. Lett., vol. 5, no. 4, pp. 135-137, Apr. 2001.

[7] M. R. D. Rodrigues and I. J. Wassell, "IMD reduction with SLM and PTS to improve the error-probability performance of nonlinearly distorted OFDM signals," IEEE Trans. Veh. Technol., vol. 55, no. 2, pp. 537-548, Mar. 2006.

[8] S. H. Han and J. H. Lee, "An overview of peak-to-average power ratio reduction techniques for multicarrier transmission," IEEE Wireless Commun., pp. 56-65, Apr. 2005.

[9] R. A. Horn and C. R. Johnson, Matrix Analysis. 1st ed. Cambridge, MA: Cambridge Univ. Press, 1985. 